\title{
RECENT EXPERIENCE WITH INDUCTIVE INSERT AT PSR
}

\author{
K.Y. Ng, D. Wildman, M. Popovic, FNAL, Batavia, IL 60510, USA \\ A. Browman, D. Fitzgerald, R. Macek, M. Plum, and T. Spickermann, \\ LANL, Los Alamos, NM 87545, USA
}

\section{Abstract}

In a Fermilab-Los Alamos collaboration, inductances constructed of ferrite cores sufficient to cancel a large fraction of the space charge potential-well distortion were installed in the Los Alamos Proton Storage Ring (PSR) as one means of raising the threshold for the two-stream e-p instability. When operating at higher intensities and with sufficient inductance added for full space-charge compensation, an unacceptable longitudinal self-bunching, microwavelike, instability was encountered. Heating the cores to $\sim 130^{\circ} \mathrm{C}$ proved to be an effective cure, and was found to be a means for tuning the inductance over a limited but useful range. The heated inductors were an essential ingredient in achieving a record accumulation of $9.7 \mu \mathrm{C} /$ pulse. An engineered version of the inductors is now installed for routine operation of the PSR. A summary of the inductor characteristics, theory of operation, experimental results, and interpretation will be presented.

\section{LONGITUDINAL INSTABILITY}

Inductive tuners were installed into the PSR to cancel the space charge effects of the intense proton beam at $799 \mathrm{MeV}$. Each tuner consists of a stainless steel pillbox cavity closely packed with 30 Toshiba $\mathrm{M}_{4} \mathrm{C}_{21 \mathrm{~A}}$ ferrite cores, each $2.54 \mathrm{~cm}$ thick, $12.7 \mathrm{~cm}$ I.D. and $20.3 \mathrm{~cm}$ O.D., and wrapped with an 88-turn solenoid. When two tuners were installed in 1997 with the intention of $2 / 3$ spacecharge compensation, clear and consistent evidence was observed, including shorter bunch length, cleaner bunch gap, and smaller rf voltage required for stable operation. After an upgrade when 3 tuners were installed in 1999 and the beam intensity was raised, a longitudinal instability was observed. Figure 1 shows a chopped coasting beam accumulated for $125 \mu \mathrm{s}$ and stored for $500 \mu \mathrm{s}$ recorded at a wide-band wall current monitor. The ripples at the beam profile indicate a longitudinal microwave-like instability at 72.7 MHz, which is roughly the resonant frequency of the pill-box cavities housing the ferrite cores. The resonance also showed up as ripples at the rear half of a $250 \mathrm{~ns}$ (left)

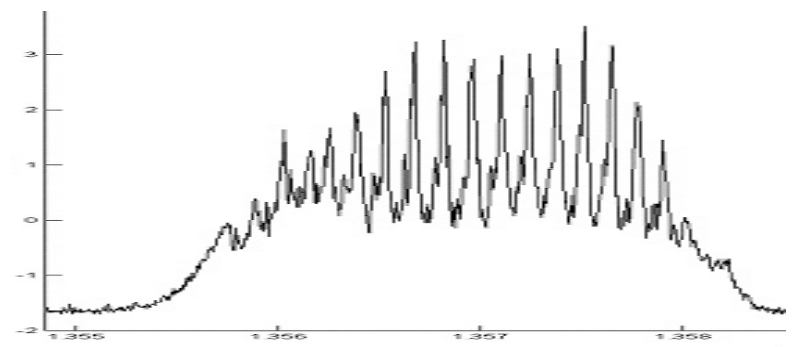

Figure 1: Longitudinal microwave-like instability recorded by wall-gap monitor of a coasting beam driven at $72.7 \mathrm{MHz}$.

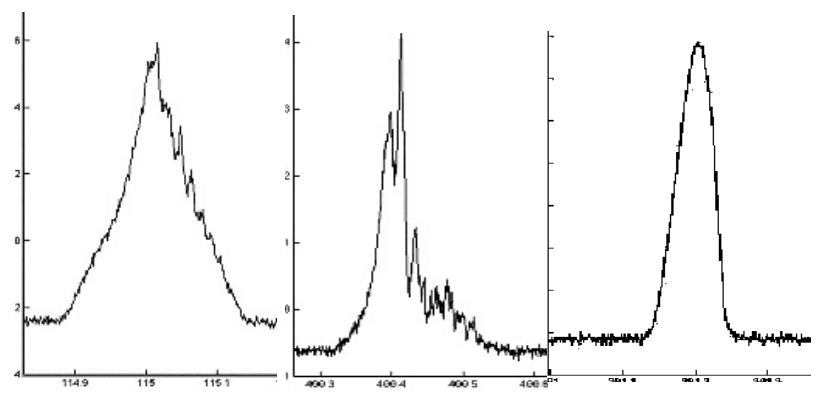

Figure 2: Instability perturbation on profiles of bunches with full width $250 \mathrm{~ns}$ (left) and $100 \mathrm{~ns}$ (middle). Right: Upon heating the ferrite to $130^{\circ} \mathrm{C}$ (see Sec. 4), the profile of the $100 \mathrm{~ns}$ bunch is no longer distorted.

and $100 \mathrm{~ns}$ bunch (middle) in Fig. 2. Apparently, the instability is tolerable for the $250 \mathrm{~ns}$ bunch because the bunch shape distortion is small. However, the $\sim 100 \mathrm{~ns}$ bunch is totally disastrous because it was lengthened to $200 \mathrm{~ns}$.

\section{CAUSE OF INSTABILITY}

To incorporate loss, the relative permeability of the ferrite can be made complex: $\mu \rightarrow \mu^{\prime}+i \mu^{\prime \prime}$. The impedance of the ferrite is therefore

$$
Z_{0}^{\|}=-i\left(\mu^{\prime}+i \mu^{\prime \prime}\right) \omega L,
$$

where $L$ denotes the inductance of the ferrite and $\omega$ the angular frequency. It is clear that $\mu^{\prime}$ and $\mu^{\prime \prime}$ must be frequency-dependent. With the ferrite cores enclosed in a pill-box cavity, the impedance can be represented by

$$
Z_{0}^{\|}(\omega)=\frac{R}{1-i Q\left(\omega / \omega_{r}-\omega_{r} / \omega\right)},
$$

where $\omega_{r}$ is roughly where $\mu^{\prime \prime}$ peaks. If we denote $\mu_{L}^{\prime}$ as the value of $\mu^{\prime}$ at low frequencies and $\mu_{R}^{\prime \prime}$ the value of $\mu^{\prime \prime}$ at resonant frequency $\omega_{r} /(2 \pi)$, we readily obtain $\mu_{R}^{\prime \prime}=$ $Q \mu_{L}^{\prime}$. Note that $Q$ here is the quality factor describing the $\mu^{\prime \prime}$ peak and is not the usual industrial-quoted $Q$. For a space-charge dominated beam, the actual area of beam stability in the complex $Z_{0}^{\|} / n$-plane (or the traditional $U^{\prime}-V^{\prime}$ plane), where $n$ is the revolution harmonic, is somewhat different from the commonly quoted Keil-Schnell estimation [2]. In Fig. 3, the heart-shape solid curve 1 is the threshold for parabolic distribution in momentum spread, where the momentum gradient is discontinuous at the ends of the spread. Instability develops and a smooth momentum gradient will result, changing the threshold curve to that of a distribution represented by 2 . Further smoothing of the momentum gradient at the ends of the spread to a Gaussian distribution will change the threshold curve to 3 . On the other hand, the commonly known Keil-Schnell criterion is denoted by the circle of unit radius in dots. This is why in many low-energy machines the Keil-Schnell limit has been significantly overcome by a factor of about 5 to 10 . 


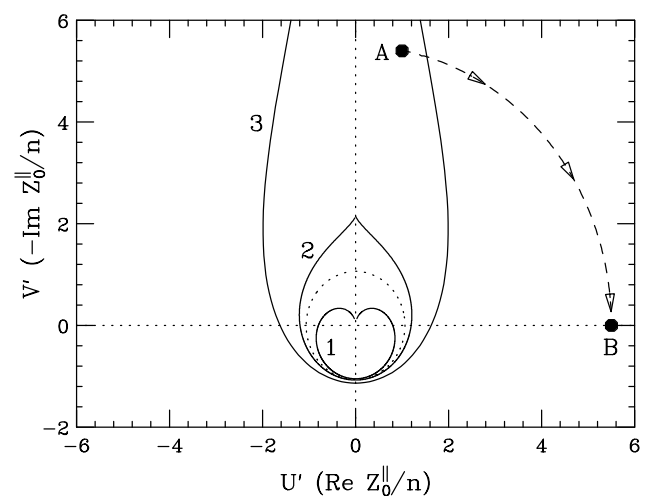

Figure 3: An intense space-charge beam may have impedance at A outside the Keil-Schnell circle (dots) but is stable inside the stability curve 3 for Gaussian distribution. Ferrite inserts compensating the space charge completely will lead to a resistive impedance roughly at B and therefore introducing instability.

Here, the space charge is almost the only source of the impedance, $\operatorname{Re} Z_{0}^{\|} / n$ typically orders of magnitude smaller. As an example, if the impedance of the Los Alamos PSR is at $\mathrm{A}$, the beam is within the microwave stable region if the momentum spread is Gaussian like, although it exceeds the Keil-Schnell limit. Now, if we compensate the spacecharge potential-well distortion by the ferrite inductance, the ferrite required will have an inductive impedance at low frequency equal to the negative value of the space charge impedance at $\mathrm{A}$, for example, about -5.5 units according to Fig. 3. However, the ferrite also has a resistive impedance or $\operatorname{Re} Z_{0}^{\|} / n$ coming from $\mu^{\prime \prime}$. Although $\operatorname{Re} Z_{0}^{\|} / n$ is negligible at low frequencies (for example, the $\mathrm{rf}$ frequency of $2.796 \mathrm{MHz}$ of the PSR), it reaches a peak value near $\omega_{r} /(2 \pi)$ (about 50 to $80 \mathrm{MHz}$ for the Toshiba $\mathrm{M}_{4} \mathrm{C}_{21 \mathrm{~A}}$ inside the pill-box container) with the peak value the same order of magnitude as the low-frequency $\operatorname{Im} Z_{0}^{\|} / n$.

Thus the ferrite will contribute a resistive impedance denoted roughly by B ( $\sim 5.5$ units) when $Q \sim 1$. This resistive impedance of the ferrite will certainly exceed the threshold curve of any momentum distribution and we believe that the longitudinal instability observed at the PSR is a result of this consideration. In order to avoid this instability, we must search for a ferrite sample having a small $\mu_{R}^{\prime \prime}$ and a large $\mu_{L}^{\prime}$, or an inductive insert with a small $Q$.

\section{HEATING THE FERRITE}

Past experience indicates that when a piece of ferrite is heated up, $\mu^{\prime}$ will increase and $\mu^{\prime \prime}$ will decrease, thus having exactly the same properties we are looking for. A measurement has been made by sending a sinusoidal wave from one end of the ferrite tuner via an antenna and receiving it with an antenna at the other end, while the loss is recorded as a function of frequency of the wave. The results are shown in Fig. 4 and reveal that the $\mu^{\prime \prime}$ resonant peak drops by a factor of about 6 when the ferrite cores are heated from the room temperature of $23^{\circ} \mathrm{C}$ to $100^{\circ} \mathrm{C}$.

The properties of the heated ferrite can be understood as follows. A piece of ferrite consists of domains with magnetization. The total magnetization is the vector sum of the magnetization of the domains. As the temperature increases, the domain magnetizations are freer to move. They tend to line up resulting in higher magnetic permeability $\mu^{\prime}$, which is what we has been observing. If the temperature becomes too high, however, the spins of individual atoms or molecules inside a domain become random and the total magnetization will drop and reach zero at the Curie temperature.

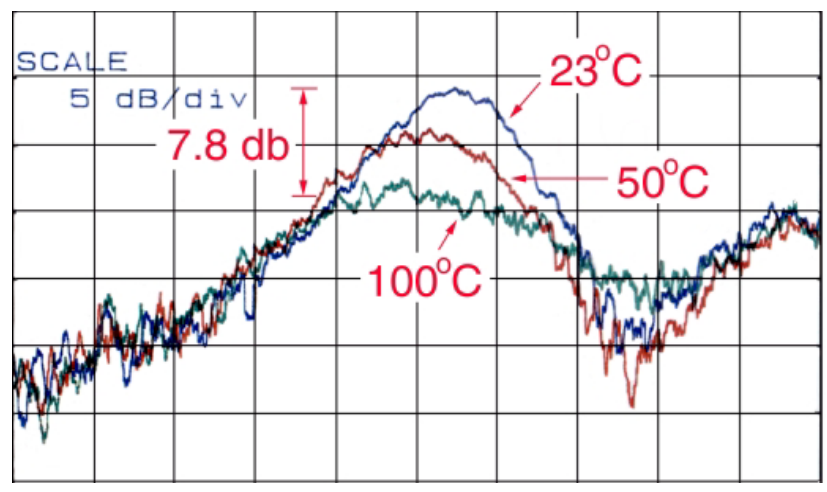

Figure 4: (color) As the ferrite cores are heated from room temperature to $100^{\circ} \mathrm{C}$, the loss has reduced by almost 6 times. Abscissa: center 75.0 MHz, span 149.4 MHz. Ordinate: $5 \mathrm{db} / \mathrm{div}$.

\section{APPLICATION AT THE PSR}

The inductive inserts have proven beneficial in raising the threshold for the two-stream e-p instability at PSR. This is readily apparent in the threshold intensity curves (plot of beam intensity at instability threshold as a function of rf buncher voltage) shown in Fig. 5. The data labeled "Inductors In" in Fig. 5 were taken just before the three ferrite inductor modules were removed and the data labeled "Inductors Out" were collected shortly thereafter. The inductance of the 3-module ferrite insert was designed to be the amount estimated to fully compensate longitudinal space charge. Later in 1999 the solenoids of the ferrite inserts for PSR were removed, the outside of the inserts were wound with heating tapes, and two modules were reinstalled in the PSR. When the ferrite is heated to $130^{\circ} \mathrm{C}$, the longitudinal microwave instability, seen in the right-hand plot in Fig. 2, disappears. The profile of the $100 \mathrm{~ns}$ bunch in the pres-

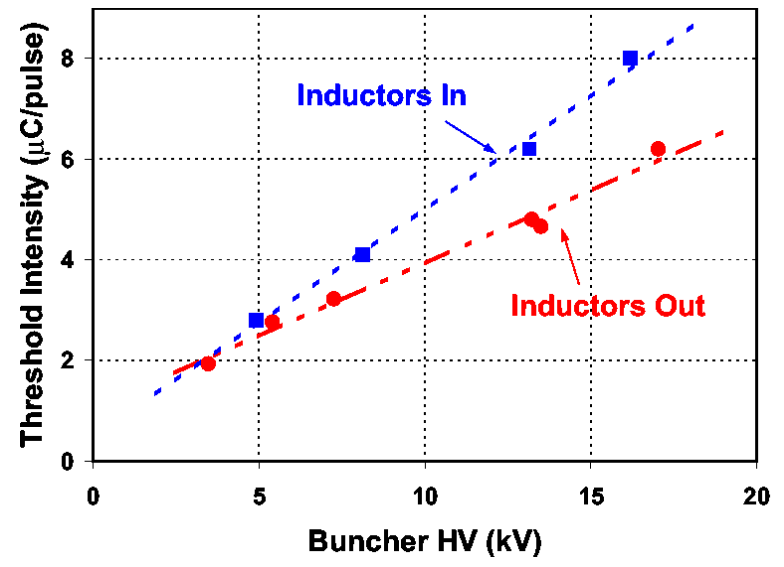

Figure 5: (color) Instability threshold intensity versus rf buncher voltage with and without the 3 -module inductive inserts. 


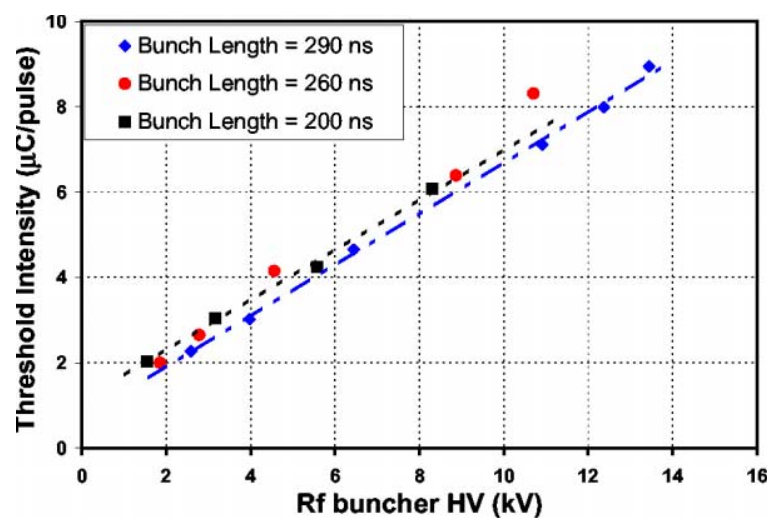

Figure 6: (color) Instability threshold intensity curves for three values of the bunch length.

ence of the heated ferrite tuners, is no longer distorted and the bunch has not been lengthened. Further beam studies with the heated ferrites carried out during the remainder of 1999 demonstrated other benefits of the inductors without unmanageable operational impacts. The gap in the beam between successive turns appears "clean"; i.e., very flat gap with more sharply defined edges and no evidence of beam leaking into the gap. This is a qualitative observation that has not yet been quantified. In addition, the inductors permit lengthening the bunch from $250 \mathrm{~ns}$ to $290-300 \mathrm{~ns}$ without encountering the instability. In fact, data taken recently and shown in Fig. 6 demonstrates that the instability threshold curves are unaffected by bunch length variations from 200 to $290 \mathrm{~ns}$. This effect is not completely understood, as a shorter bunch length would have smaller momentum spread (for a fixed rf voltage) and provide less Landau damping. However, a short bunch means a longer gap and less chance for electrons to survive the gap. These two effects would tend to cancel.

Two effects of the ferrite inserts are thought to contribute to improving the instability threshold. One is the effect of a cleaner gap that would trap fewer electrons during gap passage. The other is increased Landau damping by increased momentum spread from removal of the space charge depression of the bucket height. The latter is illustrated in Fig. 7 which shows plots from ACCSIM simulations [3] of the effect of longitudinal space charge on the rf bucket height and momentum spread for a beam of $7.3 \mu \mathrm{C} /$ pulse with $13 \mathrm{kV}$ rf voltage. Shown are the simulations (a) with and (b) without longitudinal space charge (equivalent to full compensation by inductive inserts). For this case, the space-charge effect reduces the bucket height by $\sim 23 \%$. In the absence of space charge, the bucket height scales by the square root of the rf voltage and would imply a reduction $\sim 41 \%$ in rf voltage to reach the same bucket height as with space charge. This argument implies that with inductors a $\sim 41 \%$ reduction in rf voltage would reach the same momentum spread as obtained in their absence. This is in reasonable agreement with the observed effect of $\sim 35 \%$. Thus, it appears that Landau damping explains much of the effect of the ferrite inserts on the instability.

Comparable reductions in threshold curves have been ob-

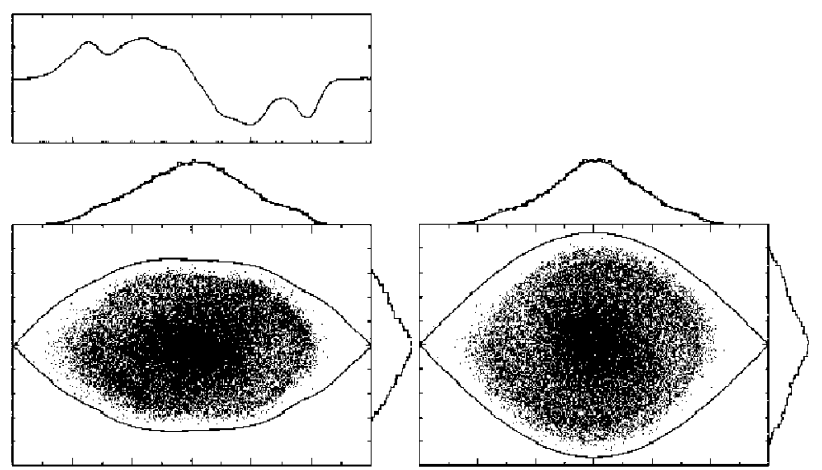

(a) Without Space Charge $(7.3 \mu \mathrm{C})$

(b) With Space Charge $(7.3 \mu \mathrm{C})$

Figure 7: Plots of results of ACCSIM simulations (a) with and (b) without longitudinal space charge. The top curve in (a) shows the space charge voltage per turn (proportional to the spatial derivative of the proton line density).

tained with other means of Landau damping such as the use of a skew quadrupole (coupled Landau damping), sextupoles and octupoles. It has also been observed that the effects of these (on the instability threshold) add with that of the inductors. In late 1999 the combined effect of heated ferrites and a skew quad enabled us to accumulate and store at the PSR a record $9.7 \mu \mathrm{C} /$ pulse, which is all that the linac could deliver. For this demonstration, the accumulation time was $1225 \mathrm{~ms}$, the maximum obtainable at $1 \mathrm{~Hz}$ from the linac. The ferrite inserts were heated to $190^{\circ} \mathrm{C}$, which over compensates longitudinal space charge by $\sim 50 \%$. The rf buncher was at the maximum of $18 \mathrm{kV}$. In addition, the bunch width was stretched out to $305 \mathrm{~ns}$, something never been accomplished before without reducing the threshold intensity. Beam losses were high $(\sim 5 \%)$, which would be prohibitive at $20 \mathrm{~Hz}$. There was, no doubt, significant emittance growth that could be attributed to transverse space charge effects from the very high peak beam current of $82 \mathrm{~A}$ observed in this demonstration. Engineered versions of the heated ferrites were installed in the fall of 2000 and have been used in production running ever since. A bunch length of $290 \mathrm{~ns}$ instead of the 250 has reduced the accumulation time accordingly thereby saving $\sim \$ 15 \mathrm{k} /$ month in linac power costs. At the present, the PSR with two heated-ferrite modules can operate stably at an intensity of $8 \mu \mathrm{C} /$ pulse for low repetition rates (for beam studies and single pulse users). Thus, the peak intensity goal of the upgrade has been surpassed. The remaining challenge is to reduce beam losses so that routine operation at $20 \mathrm{~Hz}$ is possible with acceptable activation of the ring.

\section{REFERENCES}

[1] M.A. Plum, et. al., Phys. Rev. ST Accel. Beams, 2, 064201 (1999).

[2] E. Keil and W. Schnell, CERN Report TH-RF/69-48 (1969); V.K. Neil and A.M. Sessler, Rev. Sci. Instr. 36, 429 (1965). D. Boussard, CERN Report Lab II/RF/Int./75-2 (1975).

[3] F.W. Jones, G.H. Mackenzie, and H. Schönauer, Proc. 14th Int. Conf. High Energy Accel, Part. Accel. 31, 199 (1990). 